Revue québécoise de psychologie

Revue ${ }_{\text {québoìse }}^{\text {Récol }}$

de psychologie

\title{
LES CONFLITS SÉVÈRES DE SÉPARATION : LE POINT DE VUE ET LES BESOINS DES INTERVENANTS EN PROTECTION DE LA JEUNESSE
}

\section{FAMILIES INVOLVED IN HIGH-CONFLICT SEPARATION: VIEWS AND NEEDS OF CHILD PROTECTION WORKERS}

\author{
Élisabeth Godbout, Michael Saini et Catherine Turbide
}

Volume 39, numéro 3, 2018

PSYCHOLOGIE LÉGALE : ENJEUX ÉPISTÉMIQUES, MÉTHODOLOGIQUES ET DÉONTOLOGIQUES

LEGAL PSYCHOLOGY: EPISTEMIC, METHODOLOGICAL AND

ETHICAL CHALLENGES

URI : https://id.erudit.org/iderudit/1058186ar

DOI : https://doi.org/10.7202/1058186ar

Aller au sommaire du numéro

Éditeur(s)

Revue québécoise de psychologie

ISSN

2560-6530 (numérique)

Découvrir la revue

Citer cet article

Godbout, É., Saini, M. \& Turbide, C. (2018). LES CONFLITS SÉVÈRES DE SÉPARATION : LE POINT DE VUE ET LES BESOINS DES INTERVENANTS EN PROTECTION DE LA JEUNESSE. Revue québécoise de psychologie, 39(3), 99-124. https://doi.org/10.7202/1058186ar
Résumé de l'article

Les familles aux prises avec des conflits sévères de séparation (CSS) représentent un défi de taille pour les services de protection de la jeunesse. Cet article rend compte de l'expérience d'intervenants québécois $(n=309)$ dans ce type de dossiers à l'aide d'un sondage en ligne. Les résultats révèlent que cette problématique est mal définie selon les intervenants et que le mandat de la protection de la jeunesse manque de clarté dans ces dossiers. Une approche à la fois intégrée et différenciée des services psychojudiciaires est discutée pour le développement futur de l'intervention dans ce domaine. 


\title{
LES CONFLITS SÉVĖRES DE SÉPARATION : LE POINT DE VUE ET LES BESOINS DES INTERVENANTS EN PROTECTION DE LA JEUNESSE ${ }^{1}$
}

FAMILIES INVOLVED IN HIGH-CONFLICT SEPARATION: VIEWS AND NEEDS OF CHILD PROTECTION WORKERS

\author{
Élisabeth Godbout ${ }^{2}$ \\ Université du Québec à Trois-Rivières \\ Catherine Turbide \\ Université Laval
}

Michael Saini

Université de Toronto

La séparation parentale est une réalité répandue chez les jeunes québécois et canadiens. Si la grande majorité d'entre eux s'adapte bien à cette transition, un sous-groupe d'enfants vit des difficultés plus importantes et persistantes (Amato, 2003; Hetherington, Bridges et Insabella, 1998). À cet égard, il existe un consensus voulant que le conflit entre les parents soit l'un des prédicteurs les plus significatifs des problèmes d'adaptation chez l'enfant (Amato et Keith, 1991; Emery, 1999; Grych, 2005). Les conflits les plus sérieux et persistants entre les ex-conjoints sont fréquemment nommés « conflits sévères de séparation (CSS) » ${ }^{3}$ par les professionnels des domaines psychosociaux et du droit de même que par les chercheurs (Birnbaum et Bala, 2010). II est aujourd'hui largement reconnu que les enfants exposés à des CSS sont à risque de développer de graves difficultés d'adaptation, au point où cette situation est considérée comme une forme de mauvais traitement psychologique (MTP) et un motif de compromission en protection de la jeunesse (Fauteux, 2013; Malo, 2013; Saini, Black, Fallon et Marshall, 2013). Depuis leur inclusion implicite dans la Loi sur la protection de la jeunesse en 2007, peu de travaux se sont penchés sur la réalité des intervenants en protection de la jeunesse québécoise qui doivent composer avec ces situations délicates, et ce, malgré le fait que les quelques témoignages recueillis sur le terrain indiquent que ces professionnels vivent des difficultés importantes (Malo, 2013; Malo et Rivard, 2013).

Dans le but de mieux comprendre le point de vue et les besoins des intervenants en protection de la jeunesse qui sont confrontés à des CSS dans les dossiers dont ils ont la charge, cette recherche exploratoire a pour objectifs de : saisir quelle(s) définition(s) des CSS est ou sont utilisée(s) par

1. Cette recherche a obtenu un soutien financier du Conseil de recherches en sciences humaines du Canada

2. Adresse de correspondance : Département de psychologie, Université du Québec à TroisRivières, C.P. 500, Trois-Rivières (QC), GA 5H7. Courriel : elisabeth.godbout@uqtr.ca.

3. Dans plusieurs écrits, les CSS sont aussi appelés : séparations hautement conflictuelles ou high-conflict separation or divorce. 
ces intervenants, de décrire leur expérience de travail auprès des familles vivant des CSS de même que leur point de vue sur l'organisation des services de protection destinés à ces familles et, enfin, de dégager des pistes de solution pour l'amélioration de l'intervention en protection de la jeunesse dans ces cas précis.

\section{LES CSS : DÉFINITIONS ET PRÉVALENCE}

Le terme CSS est de plus en plus utilisé, tant dans les écrits scientifiques que dans les décisions judiciaires. Des recherches par motsclés dans des bases de données en droit et en sciences sociales à partir des années 1980 et 1990 montrent une augmentation très marquée des publications et jugements qui font référence aux CSS à partir du tournant des années 2000 (Birnbaum et Bala, 2010; Turbide et Saint-Jacques, 2017). Malgré une popularité accrue de l'utilisation de ce concept, aucune définition des CSS ne fait actuellement l'unanimité. Ce terme est plutôt vu comme un fourre-tout qui regroupe différents phénomènes de sévérité variable et pouvant ou non être cooccurrents : les situations qui sont plus lourdement judiciarisées, celles où les interactions des parents sont marquées par la méfiance, la colère et l'incapacité à communiquer ou à s'entendre, les cas d'aliénation parentale de même que ceux impliquant des éléments de violence conjugale (Birnbaum et Bala, 2010). À ce propos, des auteurs invitent à utiliser une approche différenciée de la violence conjugale, c'est à dire basée sur une typologie comme celle élaborée par Johnson (Johnson, 2008). Ils insistent donc sur les distinctions importantes à faire entre la violence susceptible d'avoir cours entre les ex-conjoints qui vivent une séparation hautement conflictuelle (violence bidirectionnelle liée à l'escalade des conflits dans le couple) et la violence plus grave de type " terrorisme intime » qui comporte généralement (mais pas toujours) des actes violents plus sérieux et un déséquilibre de pouvoir entre les exconjoints (Anderson, Anderson, Palmer, Mutchler et Baker, 2010; Jaffe, Johnston, Crooks et Bala, 2008; Johnston, 2006). En outre, pour qualifier ces conflits de sévères, plusieurs auteurs considèrent qu'ils doivent notamment être encore actifs malgré le passage du temps (Anderson et al., 2010; Levite et Cohen, 2012; Johnston, Roseby et Kuehnle, 2009).

En 2012, le groupe de réflexion sur l'aliénation parentale du Centre jeunesse de Montréal-Institut universitaire se centrait sur la place de l'enfant dans le conflit et établissait que les CSS étaient une :

organisation relationnelle entre membres d'une famille nucléaire, qui se met en place avant, pendant et après la séparation conjugale et qui se caractérise par la participation de chacun des parents, simultanément ou en alternance, au dénigrement persistant et à la disqualification de l'autre parent, au point d'en devenir insensible aux besoins de l'enfant. Cette situation a pour conséquence l'instrumentalisation de l'enfant dans le conflit 
de ses parents et l'entretien de son conflit de loyauté pouvant entraîner des effets. (Malo et Rivard, 2013, p. 4)

Des auteurs ont toutefois remarqué que pareils conflits ne sont parfois alimentés que par un seul parent (Friedman, 2004; Kelly, 2003) et qu'ils sont souvent accompagnés d'autres problèmes tels les problèmes de santé mentale et troubles de la personnalité, l'abus de substance, les allégations de maltraitance envers les enfants et l'historique de criminalité d'un parent (Johnston, 1994; Saini et Birnbaum, 2007). Ces éléments compliquent d'autant plus le portrait des CSS. Chez des juges de la Cour supérieure du Québec et des experts (psychologues et travailleurs sociaux) mandatés pour faire des recommandations sur la garde et les droits d'accès, ces cas sont généralement décrits comme étant ceux où l'enfant est au cœur d'un conflit chronique entre ses parents qui n'arrivent pas à communiquer et qui éprouvent beaucoup de rancœur l'un envers l'autre. Cependant, la limite qui sépare les conflits sévères des autres conflits considérés " normaux » n'est apparue ni claire ni constante d'un professionnel à l'autre (Godbout, Parent et Saint-Jacques, 2016).

\section{Prévalence dans la population et devant les tribunaux}

En raison de difficultés dans sa définition et conceptualisation, la prévalence des CSS est difficile à établir. De plus, celle-ci a été évaluée sur la base de données de natures différentes. D'abord, quelques résultats de recherche sont basés sur des échantillons populationnels. Des recherches menées aux États-Unis montrent que de $25 \%$ à $35 \%$ des parents séparés ont une coparentalité caractérisée par un manque de coopération et des conflits élevés (Drapeau, Tremblay, Cyr, Godbout et Gagné, 2008). Les seules données populationnelles québécoises sont, à notre connaissance, celles tirées de l'Étude longitudinale du développement des enfants au Québec (ÉLDEQ, 1998-2010). Elles indiquent que $12 \%$ des mères d'enfants de six ans nés à la fin des années 1990 qui se sont séparées disent que le climat avec leur ex-conjoint est mauvais ou très mauvais (Desrosiers et Simard, 2010). Peu de données permettent ainsi d'estimer la présence des CSS au sein de la population québécoise de façon précise.

Par ailleurs, des données provenant de tribunaux familiaux montrent que certains parents utilisent plus fortement l'appareil judiciaire (c.-à-d. vont à procès pour la question de la garde ou de la pension alimentaire, se retrouvent en Cour à répétition) et sont donc plus susceptibles d'être très conflictuels (Bala, Birnbaum et Martinson, 2001). De fait, dépendamment des sources de données, on estime que de $8 \%$ à $15 \%$ des dossiers de séparation et divorce vont à procès principalement en raison d'un litige de garde et de droits d'accès, mais que la majeure partie de ceux-ci sont réglés pendant les procédures et que seuls $1,5 \%$ à $3 \%$ des dossiers sont ultimement tranchés par un juge (Biland et Schütz, 2012; 2013; Joyal, 
Lapierre-Adamcyk, Le Bourdais et Marcil-Gratton, 2002; Maccoby et Mnookin, 1992; Poitras, Cyr et Chatigny, accepté). Cependant, cette mesure de la prévalence des CSS est imparfaite : les parents qui vivent de hauts niveaux de conflits judiciaires sont, il est vrai, plus susceptibles d'être hostiles l'un envers l'autre et d'avoir une coparentalité discordante, mais ces différents groupes ne se recoupent pas parfaitement (Maccoby et Mnookin, 1992).

Quelques travaux indiquent que les dossiers comportant des CSS se retrouvent véritablement à la jonction de différentes branches du droit familial. Une recherche par mots-clés menée dans une base de données judicaires (Westlaw) a permis d'identifier 210 ordonnances prononcées en Ontario entre janvier 2010 et décembre 2014 qui comportaient à la fois un litige pour la garde et les droits d'accès et l'intervention des services de la protection de la jeunesse (Bala et Houston, 2015; Houston, Bala et Saini, 2017). La grande majorité de ces décisions ont été effectuées par un tribunal familial (sous la Loi sur le divorce ou sous la Children's Law Reform Act pour les enfants issus de couples non mariés) alors qu'une minorité de dossiers ont été décidés sous la Loi sur les services à l'enfance et à la famille (Ontario Child and Family Services Act), soit en protection de la jeunesse. II est donc possible de conclure que les services de protection de la jeunesse sont intervenus dans ces dossiers en raison d'allégations d'abus, de négligence, d'exposition à la violence conjugale et d'aliénation parentale sans avoir pour autant été retenus ou pris en charge par ces services. D'autres études (Brown, 2003; Brown, Frederico, Hewitt et Sheehan, 2000) indiquent que la proportion de dossiers où il y a des allégations de maltraitance croît à mesure que le conflit judiciaire entourant la garde perdure dans le temps et implique une multiplication des procédures. Ainsi, ces familles aux prises avec une séparation très conflictuelle sont susceptibles de se retrouver à la jonction entre le système de droit familial traditionnel et les services de la protection de la jeunesse, ce qui complexifie et alourdit leur parcours dans les services, notamment lors de la prise en charge des CSS par l'État quand le développement ou la sécurité d'un enfant est compromis.

\section{Prévalence en protection de la jeunesse}

Au sein même des services de protection de la jeunesse, des données ont été colligées sur la présence des CSS. Deux cas de figure sont possibles pour expliquer la trajectoire de ce problème dans ces services. Certains de ces dossiers sont directement signalés pour MTP liés à l'exposition aux CSS ou encore des situations sont signalées en raison d'une ou de plusieurs autres formes de maltraitance et ces allégations (fondées ou non) masquent des CSS qui sont découverts après l'investigation (Houston et al., 2017).

Des données indiquent que les litiges entourant la garde ou les droits d'accès et dont le tribunal est saisi (qui accompagnent régulièrement les 
CSS) seraient présents dans $12 \%$ des dossiers évalués en protection de la jeunesse au Canada (excluant le Québec) (Saini et al., 2013). Ces cas se distingueraient des autres dossiers de protection par des réouvertures plus fréquentes des dossiers (plus de trois fois), par des taux plus élevés de signalements considérés fallacieux ou fabriqués de façon intentionnelle (parmi les cas jugés non fondés) et par un nombre accru de problèmes de fonctionnement chez les enfants concernés (Saini et al., 2013). L'analyse de dossiers ontariens de protection de la jeunesse en 2013 (Ontario Incidence Study of Reported Child Abuse and Neglect) montre que $12 \%$ d'entre eux comportaient un litige de garde ou de droits d'accès (ce qui est équivalent au taux observé dans l'échantillon canadien). Dans cet échantillon, la présence d'un conflit de garde était significativement associée à la non-rétention du signalement pour poursuite des services (l'équivalent québécois est de considérer que la sécurité et le développement de l'enfant ne sont pas compromis même si le signalement est jugé fondé) (Deljavan, Saini, Fallon et Black, 2016), et ce, alors même que ce type de dossiers est plus susceptible d'être rouvert plusieurs fois (Saini et al., 2013). Ainsi, loin de se régler avec le passage du temps, ces dossiers risquent de se retrouver à répétition dans les services de protection de la jeunesse et des chercheurs et praticiens craignent que leur fermeture prématurée contribue à leur aggravation au fil du temps (Black et al., 2016; Brown, 2003).

Les données québécoises disponibles sont, quant à elles, fondées sur une analyse des différentes sous-catégories du motif de compromission référant aux MTP. Selon le bilan des directeurs de la protection de la jeunesse en 2015-2016, 15,7 \% des évaluations faites par les services de protection de la jeunesse ont été effectuées en raison de MTP. De ce nombre, $14,8 \%$ comportaient des conflits de séparation. Ce motif de compromission arrive en deuxième position en importance puisque ce sont les situations d'exposition à la violence conjugale qui représentent la forme de MTP la plus fréquemment signalée avec une proportion de $51,8 \%$ des dossiers (Institut national d'excellence en santé et en services sociaux [INESSS], 2016). En 2016-2017, 16,6 \% des signalements étaient retenus pour MTP et environ $19 \%$ d'entre eux l'étaient en raison de l'exposition des enfants aux CSS, ce qui représente la situation d'environ 1136 enfants à l'échelle du Québec (Charette, 2018; Ministère de la Santé et des Services sociaux, 2017). Bien que ces cas puissent sembler très minoritaires sur l'ensemble des dossiers, ils mobilisent un temps d'intervention disproportionné (Saini et al. 2012). À ce propos, la façon dont ces dossiers ont été traités par les intervenants en protection de la jeunesse a évolué au fil du temps, et ce, en fonction de différents changements législatifs. 


\section{LES CHANGEMENTS LÉGISLATIFS ET LEURS CONSÉQUENCES POUR LE TRAVAIL DES INTERVENANTS}

Le premier changement législatif majeur ayant fortement influencé les pratiques entourant la prise en charge des CSS au Québec est, sans conteste, la réforme de la Loi sur la protection de la jeunesse entrée en vigueur en 2007. Parmi les modifications apportées, l'ajout d'un motif de compromission référant explicitement aux MTP a ouvert la possibilité pour le DPJ4 de reconnaître des situations pouvant affecter les enfants qui avaient pu passer sous le radar des services par le passé. C'est sous l'impulsion de travaux de recherche (Gagné, 2001; Gagné et Bouchard, 2000) que les MTP ont été conceptualisés comme une forme de mauvais traitement spécifique et qu'on a recommandé leur intégration dans la Loi (Comité d'experts sur la révision de la Loi sur la protection de la jeunesse, 2004). Bien qu'on reconnaisse qu'il soit plus difficile de préciser les critères permettant d'établir la compromission sur la base des conséquences négatives de ce type de mauvais traitements sur l'enfant, le libellé de la Loi s'est centré sur un risque potentiel basé sur l'observation de comportements susceptibles de causer un préjudice à l'enfant plutôt que sur l'observation de ses conséquences chez l'enfant, celles-ci pouvant être différées dans le temps ou découler d'autres formes de maltraitance (Malo, 2014). Dans ce nouveau cadre, les CSS ont rapidement été considérés comme une forme de MTP (Malo et Rivard, 2013), et ce, bien qu'ils ne soient pas spécifiquement nommés dans la Loi sur la protection de la jeunesse :

[...] lorsque l'enfant subit, de façon grave ou continue, des comportements de nature à lui causer un préjudice de la part de ses parents ou d'une autre personne et que ses parents ne prennent pas les moyens nécessaires pour mettre fin à la situation. Ces comportements se traduisent notamment par de l'indifférence, du dénigrement, du rejet affectif, du contrôle excessif, de l'isolement, des menaces, de l'exploitation, entre autres si l'enfant est forcé à faire un travail disproportionné par rapport à ses capacités, ou par l'exposition à la violence conjugale ou familiale; 5

Il est difficile d'identifier avec précision ce qui a amené les intervenants des services de la protection de la jeunesse à inclure les CSS comme une forme de MTP. Cependant, on peut supposer que le développement de la recherche sur les facteurs prédisant l'adaptation de l'enfant à la rupture parentale et sur les formes de mauvais traitements psychologiques associées à ces contextes (comme l'aliénation parentale) (Amato et Keith, 1991; Buehler et al., 1997; Cummings et Davies, 1994; Emery, 1999; Gagné et Bouchard, 2000; Gagné, Cyr, Drapeau, Saint-Jacques et Lessard, 2011; Grych, 2005; Hetherington et Stanley-Hagan, 1999; Kelly, 2000; Maccoby et Mnookin, 1992; Noller, Feeney, Sheehan, Darlington et Rogers, 2008) a

4. Dans cet article, l'acronyme DPJ signifie soit le Directeur de la protection de la jeunesse ou la Direction de la protection de la jeunesse.

5. Article 38c) de la Loi sur la protection de la jeunesse, RLRQ, c. P-34.1 (L.P.J.). 
sensibilisé les intervenants aux impacts d'une rupture conflictuelle sur le développement de l'enfant. Comme la recherche sur les CSS en protection de la jeunesse en est encore à ses débuts au Québec, il est difficile de savoir si le manque de définition précise des CSS dans la Loi sur la protection de la jeunesse (LPJ) a un impact sur la pratique des intervenants. II serait également pertinent de vérifier s'il existe des disparités dans l'interprétation de ce concept entre les juges, les avocats et les intervenants cliniques et, le cas échéant, si celles-ci influencent les orientations prises par le DPJ et la Cour.

Tel qu'abordé précédemment, sur le plan judiciaire, ces dossiers comportant des CSS sont susceptibles d'être vus par deux instances différentes dans le cas où ces conflits sont associés à la compromission de la sécurité ou du développement d'un enfant. En effet, les compétences des tribunaux en matière familiale sont partagées entre la Cour supérieure qui traite notamment des demandes relatives au divorce et à la séparation, à la garde et aux droits d'accès des enfants et à l'autorité parentale ${ }^{6}$ alors que la Cour du Québec a l'exclusivité des questions entourant la protection de la jeunesse conformément à la Loi sur la protection de la jeunesse ${ }^{7}$. Lorsque le tribunal de la jeunesse de la Cour du Québec conclut que la sécurité et le développement d'un enfant sont compromis, ce dernier possède une compétence exclusive pour décider à qui l'enfant sera confié, à qui il pourra avoir accès de même qu'il pourra se prononcer sur certains attributs de l'autorité parentale, et ce, durant une période déterminée, soit jusqu'à la fin de la situation de compromission. Cette ordonnance est donc à la fois temporaire et confidentielle et ne peut être utilisée devant d'autres instances (par exemple, Revenu Québec) et elle ne règle pas la question de la pension alimentaire qui demeure du ressort de la Cour supérieure (Perron et Labrecque Tremblay, 2015). De plus, selon Perron et Labrecque Tremblay (2015) il arriverait fréquemment que le DPJ exige que les parents clarifient leur situation matrimoniale à la fermeture de leur dossier, ce qui implique un passage devant la Cour supérieure.

Ce dédoublement du traitement judiciaire d'une même situation familiale est identifié depuis longtemps comme très exigeant en termes de temps et de ressources, tant pour les familles que pour le système de justice (Conseil de la famille et de l'enfance, 2003). Des solutions qui évitent le morcellement des interventions et permettant d'apporter une solution globale à un problème familial sont discutées depuis les années 1970, notamment par la

6. Article 33 du Code de procédure civile, RLRQ, c. C-25.01. Par ailleurs, selon la Cour d'appel du Québec : en principe, la Cour supérieure a compétence exclusive en matière de garde d'enfants et de droits d'accès. Ce n'est donc qu'à titre exceptionnel qu'un autre tribunal peut posséder une telle compétence : Droit de la famille - 1873, 1994 CanLII 5862 (QC C.A.), [1994], R.J.Q. 1787, 1789-1790 (C.A.).

7. Article 83, paragraphe 2 de la Loi sur les tribunaux judiciaires, RLRQ, C. T-16 (L.T.J.). 
constitution d'un tribunal unifié de la famille ayant compétence sur l'ensemble des affaires familiales (adoption, protection de la jeunesse, garde d'enfants, etc.) (Comité de réflexion et d'orientation sur la justice de première instance au Québec, 2005; Conseil de la famille et de l'enfance, 2003). Plus modestes, les changements au Code de procédure civile entrés en vigueur en janvier 2016 et qui font en sorte d'attribuer de nouvelles compétences à la Cour du Québec pourraient constituer un pas vers une meilleure intégration. En effet, l'article 37 alinéa 3 du Code stipule désormais que (soulignements ajoutés) :

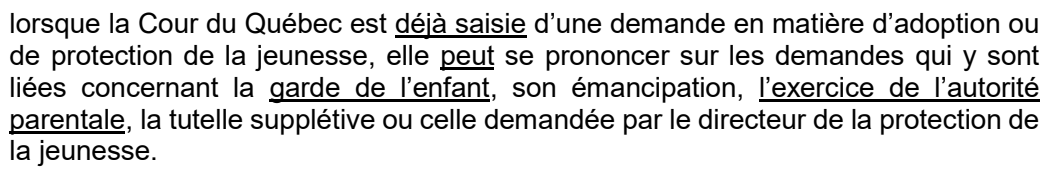
de protection de la jeunesse, elle peut se prononcer sur les demandes qui y sont liées concernant la garde de l'enfant, son émancipation, l'exercice de l'autorité parentale, la tutelle supplétive ou celle demandée par le directeur de la protection de la jeunesse.

En apportant ces changements, l'objectif du législateur était de clarifier l'état de la jurisprudence sur l'étendue de la compétence de la Cour du Québec qui était contradictoire en la matière, de favoriser l'accès à la justice, de simplifier la procédure et de diminuer les coûts (Perron et Labrecque Tremblay, 2015). Cependant, deux questions s'imposent. D'abord, ces modifications sont-elles susceptibles de répondre aux objectifs du législateur? Pour Perron et Labrecque Tremblay (2015), considérant qu'une autre demande indépendante est effectuée pour traiter de ces demandes accessoires, que la procédure est différente (procédure civile), quel sera le gain réel en termes d'efficacité? Une seconde question se pose quant aux conséquences de ces changements dans la pratique des intervenants en protection de la jeunesse. Ce second dossier de cour utilise la même enquête, soit la même preuve que le dossier de protection de la jeunesse et le DPJ est mis en cause dans cette nouvelle demande, ce qui a des implications encore méconnues pour la pratique des intervenants en protection de la jeunesse (Charette, 2018). La présente recherche ne peut répondre à ces questions puisque nos données n'ont pas été collectées alors que les changements au Code de procédure civile étaient en vigueur. II n'en demeure pas moins que le travail des intervenants auprès de ces familles en conflit doit être compris comme se situant à la jonction de ces deux domaines de compétences de la Cour du Québec et de la Cour supérieure, ce qui n'est pas sans avoir de conséquences sur le traitement de ces dossiers et les différentes pressions pouvant être exercées sur ces professionnels de la part des parents et des tribunaux. Par ailleurs, la formulation de l'article 37 du Code créerait un flou interprétatif qui devra être suivi de près, tant dans ses effets sur l'administration de la justice que dans ceux impliquant les services de protection de la jeunesse (Barreau du Québec, 2013; Charette, 2018). 


\section{ENJEUX POUR LA PRATIQUE DES INTERVENANTS}

Cette zone grise concernant le rôle des intervenants à la frontière du droit familial et du droit de la jeunesse a bien été mise en lumière dans une première recherche qualitative de Saini et ses collaborateurs (2012) basée sur les témoignages de 28 intervenants en protection de la jeunesse en Ontario. Ces résultats ont été ensuite largement confirmés lors d'un sondage mené dans cinq agences de protection de l'enfance (Houston et al., 2017). Ces intervenants ont rapporté ressentir une pression de la part du système de justice à prendre position sur la garde et ont déploré le manque de clarté de leur rôle et mandat dans ces situations qui devrait plutôt se centrer sur la compromission de la sécurité ou du développement de l'enfant (Houston et al., 2017; Saini et al., 2012). D'autres défis de l'intervention ont également été mis au jour à partir de données recueillies auprès d'intervenants : les lacunes en termes de formation spécialisée sur les CSS, l'exigence élevée en ressources, en temps et en énergie que ces dossiers représentent, les tentatives de manipulation de la part des parents et le sentiment constant d'être soi-même triangulé dans le conflit (Malo, 2013; Houston et al., 2017; Saini et al., 2012). Les intervenants doivent évaluer si les allégations sont fondées dans un contexte où elles se multiplient et où les parents auraient tendance à formuler de fausses accusations pour obtenir gain de cause (Houston et al., 2017; Jevne et Andenæs, 2017; Saini et Birnbaum, 2007; Saini et al., 2012). Ils observent aussi des problèmes de santé mentale chez ces parents qui peuvent ajouter aux défis liés à l'intervention (Fauteux, 2013; Saini et al., 2012).

Un autre défi important de l'intervention a trait au traitement des allégations de maltraitance qui sont effectuées de part et d'autre pour en arriver à prendre position sur le risque de préjudice pour l'enfant sur la base de faits observables (Saini et al., 2012). Selon plusieurs auteurs, il existe un risque réel de ne pas donner suite à ces situations par manque d'éléments tangibles pour soutenir la compromission alors que ces enfants sont en fait en besoin de protection (Deljavan et al., 2016; Platt et al., 2016). Brown (2003) et Buckley, Whelan et Carr (2011) avancent que les intervenants peuvent minimiser les allégations parce qu'elles sont faites dans un contexte de conflit et que celui qui signale (souvent l'autre parent) manque de crédibilité. II se pourrait aussi que les intervenants évaluent mal les impacts du conflit en considérant que ce dernier représente un stress temporaire prenant fin lorsque le conflit de garde est réglé (Brown, Frederico, Hewitt et Sheehan, 2001; Johnston, Lee, Olesen et Walters, 2005).

Il apparaît également crucial de bien distinguer les conflits sévères de séparation des situations de violence conjugale. Compte tenu du nombre de situations de conflits sévères de séparation où l'on constate la présence de comportements violents (Bala et Houston, 2015; Deljavan et al., 2016; Fauteux, 2013), les intervenants des services de protection doivent 
s'assurer de ne pas alimenter une dynamique où un parent utilise son pouvoir contre l'autre. Ils doivent aussi éviter de considérer une personne victime de violence conjugale comme impliquée à parts égales dans le conflit et prévenir les situations qui reproduiraient la dynamique de violence (Birnbaum et Bala, 2010; Jaffe et al., 2008; Platt et al., 2016). Des données exploratoires montrent également que des parents se retrouvent dans une situation paradoxale où ils ont l'impression que les services de protection leur reprochent de ne pas avoir quitté assez rapidement une situation de violence conjugale mettant à risque leur enfant tout en ayant de la difficulté à faire entendre leurs doléances envers leur agresseur une fois la rupture effective (Brown, 2003; Buckley et al., 2011; Platt et al., 2016).

Dans cet ordre d'idées, quelques résultats de recherche sont disponibles sur la perspective des parents aux prises avec des CSS et qui se retrouvent dans le dédale des services de protection de la jeunesse. Plusieurs parents y affirment avoir perdu toute crédibilité par rapport aux intervenants à partir du moment où ces derniers ont réalisé qu'ils étaient en situation de rupture conjugale (Buckley et al., 2011). Ainsi, le doute quant à la véracité des dires de ces parents et la suspicion quant à leurs allégations pourraient teinter l'intervention effectuée dans ces dossiers.

\section{MÉTHODOLOGIE}

La population étudiée dans la présente recherche est celle des intervenants œuvrant dans les Centres intégrés de santé et de services sociaux (CISSS) et Centres intégrés universitaires de santé et de services sociaux (CIUSSS) du Québec et dont le travail est régi par la Loi sur la protection de la jeunesse. Ces intervenants travaillent à différentes étapes de l'intervention du DPJ à la suite d'un signalement, soit: 1- réception et traitement des signalements, 2- évaluation et orientation, 3- application des mesures et 4- révision, mais des répondants ont aussi été recrutés dans les services de réadaptation pour cette clientèle ou comme spécialistes ou conseillers en activités cliniques. Un échantillon non probabiliste composé de volontaires a été formé. Au total, 309 intervenants ont répondu au moins en partie au questionnaire en ligne. La collecte des données s'est déroulée d'octobre 2015 à mars 2016 dans quatre établissements du réseau de la santé et des services sociaux, soit les CISSS Montérégie Est (39\% des répondants, $\mathrm{n}=88$ ), le CISSS du Bas-Saint-Laurent (12\% des répondants, $\mathrm{n}=26)$, le CISSS de Chaudière-Appalaches ( $11 \%$ des répondants, $n=24)$ et le CIUSSS de la Capitale-Nationale (38\% des répondants, $n=85)$. Par ailleurs, la majorité des répondants rapportent desservir la population d'un grand centre urbain $(42,8 \%)$ ou d'une ville de taille moyenne $(39,2 \%)$. Environ $18 \%$ des intervenants sondés estiment exercer en milieu rural $(3,6 \%)$ ou dans une ville de moins de 50000 habitants (14,4\%). Le profil 
détaillé des répondants est présenté au Tableau 1. Les femmes sont nettement majoritaires au sein de l'échantillon, de même que les personnes de moins de 40 ans, détentrices d'un baccalauréat et formées en service social. La plupart des intervenantes participantes sont également détentrices d'un poste et travaillent en évaluation des signalements et orientation des mesures ou encore à l'application des mesures. Enfin, la majorité a 10 ans d'expérience ou moins en protection de la jeunesse.

Les données ont été recueillies à l'aide d'un questionnaire en ligne entièrement anonyme comportant 62 questions portant sur différents aspects du travail des intervenants auprès des familles vivant des CSS : la définition des CSS, le niveau d'expérience et d'aisance à travailler avec cette clientèle, l'encadrement du milieu de travail et les approches utilisées, les facteurs pertinents à relever concernant ce problème et des pistes d'amélioration pour la pratique. Les questions sont en majorité à choix de réponses (choix entre plusieurs options et échelles Likert) et quelques questions sont ouvertes. Ces dernières sont généralement utilisées afin de préciser une réponse à une question fermée, mais elles ont également servi à demander aux répondants de définir certains concepts dans leurs propres mots ou de recueillir leur expérience et leurs commentaires. Ce questionnaire a été utilisé en Ontario (par le biais de la Ontario Association of Children's Aid Societies) (Houston et al., 2017). Le présent projet est une traduction et une adaptation au contexte québécois du projet ontarien initial. Le devis de recherche est mixte et a une finalité descriptive et exploratoire. La partie quantitative comporte des analyses statistiques de fréquence et des analyses univariées et bivariées (test du $\mathrm{khi}^{2}$ ). Les données qualitatives recueillies à l'aide des questions ouvertes et à développement ont été analysées par analyse de contenu thématique (Paillé et Mucchielli, 2008). Des extraits de ces réponses sont présentés dans la section des résultats afin d'illustrer le propos des intervenants.

\section{RÉSULTATS}

\section{Les caractéristiques des CSS du point de vue des intervenants}

Une faible majorité (53\%) des répondants $(n=301)$ sont d'avis que leur établissement a établi une définition claire du terme " conflits sévères de séparation », $50 \%$ croient que cette définition n'inclut pas la violence conjugale (32\% sont incertains et $17 \%$ croient que la violence conjugale est incluse dans cette définition) et $54 \%$ sont d'opinion que la violence conjugale ne devrait pas faire partie de la définition des CSS $(11 \%$ sont incertains et $35 \%$ croient que la violence conjugale devrait faire partie de la définition). Les 260 intervenants ayant répondu à la question ouverte: « dans vos propres mots, qu'est-ce qui définit une famille vivant des conflits sévères de séparation? » font surtout état des comportements des parents 
Tableau 1

Profil sociodémographique et professionnel des répondants

\begin{tabular}{rrrr}
\hline & & $\%$ & $(\mathrm{n})$ \\
\hline Sexe & & & \\
& Homme & 10,4 & $(23)$ \\
& Femme & 89,6 & $(199)$ \\
Âge & & & \\
& $20-29$ & 25,6 & $(57)$ \\
& $30-39$ & 47,5 & $(106)$ \\
& $40-49$ & 16,1 & $(36)$ \\
& $50-59$ & 9,9 & $(22)$ \\
& $60+$ & 0,9 & $(2)$
\end{tabular}

Domaine de formation professionnelle

Travail social/service social

Psychologie

Psychoéducation

Criminologie

58,4

7,2

(16)

Autre

Dernier diplôme obtenu

Doctorat

6,8

Maîtrise

0,0

Baccalauréat

14,8

Certificat de premier cycle

DEC professionne

73,5

(164)

Autre

5,8

Secteur

Réception et traitement des signalements 2,3

Évaluation et orientation

Application des mesures

48,2

37,4

(107)

Révision

(83)

Autre

4,1
8,1

(9)

Statut d'emploi

Temps complet - détenteur de poste

$65,1 \quad(142)$

Temps partiel - détenteur de poste

$5,1 \quad(11)$

Sur appel/contractuel - non détenteur de poste

25,7

Autre

Type d'emploi

ARH et réviseur

$81,4 \quad(180)$

Technicien en assistance sociale

Cadre

5,0

Autre (éducateur, spécialiste en activités cliniques, etc.)

Années d'expérience en protection de la jeunesse

Moins d'un an

1,4

$1-2$ ans

10,4

3-5 ans

15,8

(3)

6-10 ans

$11-15$ ans

27,6

(35)

16-20 ans

21,27

+ de 20 ans

14,0

(21) 
qu'ils jugent inadéquats : dénigrement de l'autre parent, exposition de l'enfant au conflit et à la violence, triangulation de l'enfant dans le conflit, incapacité à communiquer et à faire le deuil de la séparation, perception erronée ou déformée de la réalité et insensibilité aux besoins de l'enfant.

Les ex-conjoints sont dénigrants l'un envers l'autre. Placent les enfants en conflit de loyauté. Responsabilisent l'autre pour tous les problèmes. Ont une faible capacité d'introspection et sont envahis par la séparation. S'accompagne de violence psychologique et parfois physique. (Intervenante 265, évaluation et orientation)

Dans une moindre mesure, des répondants mentionnent que les conflits sévères de séparation ont des impacts sur les enfants sans que ceux-ci soient précisés (il est question d' "impacts négatifs », de " symptômes ", de " séquelles », de "dommages ", de "répercussions importantes », etc.). En concordance avec le libellé de la Loi sur la protection de la jeunesse, certains mentionnent la compromission de la sécurité ou du développement des enfants exposés aux conflits. Plus fréquemment, les réponses réfèrent à un enfant pris dans un conflit de loyauté ou poussé à exclure un parent.

Lorsque les enfants sont pris dans un conflit de loyauté et doivent choisir l'un de leurs parents pour se préserver (Intervenante 276, évaluation et orientation).

Enfin, la durée des conflits est un autre élément servant à qualifier les CSS. Celle-ci est le plus souvent évoquée sans en préciser la durée : il est question de conflits " chroniques », " récurrents », qui " persistent » ou qui " perdurent».

Concernant les questions relevant du droit de la famille qui sont les plus courantes dans les dossiers comportant des CSS, la garde, d'une part, et les droits d'accès, d'autre part, ont été identifiés par une très large majorité de répondants (respectivement $96 \%$ et $93 \%$ des 256 répondants qui ont répondu à cette question). La pension alimentaire pour enfant a été nommée par $69 \%$ des répondants alors que la pension alimentaire pour l'ex-conjoint ou l'ex-conjointe et le déménagement d'un parent semblent moins fréquents puisqu'ils ont respectivement été sélectionnés par près du quart des répondants.

\section{Aisance, stress et formation des intervenants}

À la question où les répondants devaient situer leur niveau d'aisance à travailler auprès des familles vivant des conflits sévères de séparation, seulement $27 \%(n=267)$ se disent plutôt $(24 \%)$ ou très à l'aise (3\%) alors que $34 \%$ se disent neutres et $39 \%$ ne se sentent pas très à l'aise (34\%) ou pas du tout à l'aise (5\%). L'aisance des répondants est significativement associée au niveau d'expérience avec ce type de dossiers $\left[X^{2}(4\right.$, 
$267)=55,18, p<0,001]$ et au nombre d'années d'expérience à la DPJ. Les intervenants ayant plus de 10 ans d'expérience sont surreprésentés parmi les intervenants plutôt à l'aise et très à l'aise d'intervenir dans ce type de dossiers, contrairement aux intervenants avec moins de 10 ans d'expérience qui sont, pour leur part, sous-représentés dans ces catégories $\left[X^{2}(4, \quad 221)=17,50, \quad p<0,01\right]$. De plus, les hommes sont proportionnellement plus nombreux que les femmes à être très à l'aise ou plutôt à l'aise avec ce type de dossiers $\left[X^{2}(2,222)=8,46, p<0,05\right]$, même si les hommes et les femmes ne diffèrent pas quant au nombre d'années de service à la $\mathrm{DPJ}\left[X^{2}(2,220)=2,10, p=0,35\right]$. À la question : " quel niveau de stress est associé aux dossiers comprenant des conflits sévères de séparation? ( $1=$ très peu stressants comparativement aux autres dossiers à $5=$ ces dossiers sont les plus stressants de tous) ", la majorité des 225 répondants qui ont répondu à cette question ont attribué un score de 4 (49\%) ou de $5(18 \%)$ et très peu (8\%) ont donné un score de 1 ou de 2 à ce type de dossiers $(M=3,76, E T=0,86)$. Par ailleurs, $94 \%(n=267)$ rapportent ne pas avoir été spécifiquement formés à travailler auprès des familles vivant des CSS.

Le contexte de travail

À la question : " est-ce que l'établissement qui vous emploie a établi une politique ou des procédures claires concernant le rôle de la protection de la jeunesse dans les cas de conflits sévères de séparation? ", la grande majorité des 259 répondants ont soit répondu que leur établissement n'avait pas établi de telles procédures $(41 \%)$ ou bien qu'ils en étaient incertains (33\%). Seuls $26 \%$ ont répondu par l'affirmative. Ces derniers sont proportionnellement plus nombreux en Montérégie que dans les autres établissements $\left[X^{2}(6,223)=17,03, p<0,01\right]$, bien qu'au sein même de cet établissement, les opinions soient très partagées. Quelques questions avaient pour objectif de situer l'opinion des intervenants concernant le soutien qu'ils reçoivent de la part de leurs supérieurs et de leur établissement. Les avis à ce sujet sont variés alors que $37 \%$ des 259 répondants croient que leur établissement offre un soutien ou une supervision adéquate aux intervenants tandis que $37 \%$ pensent le contraire et que $26 \%$ sont incertains. Cette perception n'est pas liée à l'établissement où les intervenants travaillent $\left[X^{2}(6,2)=11,55, p=0,07\right]$. Les positions sont également diverses en ce qui a trait à la perception du soutien offert par les cadres ou supérieurs immédiats relativement aux CSS : $45 \%$ jugent en recevoir suffisamment, $18 \%$ rapportent en recevoir insuffisamment, $33 \%$ croient qu'ils en reçoivent parfois suffisamment et $4 \%$ sont incertains $(n=225)$. Parmi les répondants ayant expliqué leur réponse $(n=85)$, ceux qui expriment avoir reçu un bon soutien font état de la disponibilité de leur chef de service, du travail en équipe et de la co-intervention qui permet d'échanger, de ventiler, de trouver des stratégies d'intervention et de référer 
les parents au bon endroit. Ceux ayant le sentiment d'être moins bien soutenus rapportent que leurs supérieurs peuvent banaliser la pression, la manipulation et les agressions verbales des parents. Ils mentionnent, en outre, que leurs supérieurs manquent de temps, qu'ils ne sont pas nécessairement mieux formés et font face au même manque de moyens qu'eux devant ce problème. L'analyse qualitative de ces réponses montre également qu'à mi-chemin entre ces perceptions positives et négatives, certains estiment qu'ils ne reçoivent pas plus de soutien pour ce problème que pour d'autres, qu'ils en reçoivent au besoin, mais pas d'emblée ou que ce soutien dépend du contexte (du chef de services, de l'équipe ou du dossier particulier). De plus, des intervenants sont d'avis que ces dossiers demeurent difficiles à gérer malgré le soutien.

Considérant leur charge de travail, la majorité (61\% des 256 répondants) des intervenants jugent ne pas avoir assez de temps pour répondre aux demandes des dossiers comportant des CSS, seuls $10 \%$ croient avoir assez de temps et $29 \%$ se disent neutres sur cette question. La majorité des intervenants ont également le sentiment que leur rôle est plutôt incompris (64\% des 256 répondants) ou pas du tout compris (4\%) par les professionnels d'organisations externes (par exemple, les intervenants en CLSC, ceux qui effectuent de la médiation familiale ou des expertises, les avocats, etc.) avec lesquels ils doivent collaborer. Ceci a pour conséquences que les limites de leur rôle sont mal saisies. En effet, dans les réponses à la sous-question ouverte: "qu'est-ce que ces professionnels devraient savoir au sujet du rôle des intervenants de la DPJ pour qu'il y ait une meilleure collaboration? » répondue par 232 personnes, certains thèmes sont présents. II est d'abord question des limites de leur rôle, notamment dans la prise en charge de certains dossiers ou dans le positionnement par rapport à la garde. Le cadre particulier de la LPJ qui rend nécessaire d'établir une preuve basée sur des faits vérifiables quant à la compromission de l'enfant est également nommé :

II semble souvent difficile pour les collaborateurs de comprendre la notion de « sécurité et développement compromis » en vertu de la LPJ, et que les comportements malsains d'un parent ne signifient pas nécessairement que la sécurité ou le développement est compromis, par exemple si un enfant est plus résilient, ou encore si le milieu compense pour les difficultés parentales. (Intervenante 93, application des mesures)

II faudrait que, dans un premier [temps], nous même sachions notre rôle, qu'il soit clair et qu'on ne pense pas que la DPJ peut venir tout changer au niveau de la garde légale. Nous ne sommes pas là pour prendre en charge la gestion de la garde, mais bien pour statuer sur l'impact que le conflit sévère peut avoir sur les enfants. (Intervenante 174, évaluation et orientation) 
De plus, certains intervenants restent sur l'impression que des professionnels ont une opinion sur la situation basée sur un parti pris et des informations partielles, alors que les intervenants de la DPJ ont eux-mêmes accès à l'ensemble de la situation et qu'ils ont un devoir de neutralité :

Que lorsqu'un partenaire/professionnel est en lien avec un parent et est au fait de la situation, il a beaucoup de difficulté à rester neutre. Cela éprouve notre travail parce qu'ils essaient parfois de défendre à tout prix le parent avec qui ils font affaire. (Intervenante 309, évaluation et orientation)

\title{
Les défis de l'intervention auprès des familles présentant des CSS
}

Afin d'identifier quels sont les principaux défis organisationnels et professionnels vécus par les intervenants en protection de la jeunesse qui travaillent auprès de cette clientèle, une question à choix de réponses était proposée. Parmi ces réponses, celles ayant obtenu une adhésion de plus de la moitié des intervenants $(n=258)$ sont le manque de formation $(69 \%)$, le manque de clarté du rôle des intervenants de la DPJ (54\%), le stress psychologique et émotionnel (54\%) et la difficulté à établir la preuve en Cour (51\%). Les conséquences de ces défis sont multiples et des intervenants $(n=238)$ les décrivent, en réponse à une question ouverte, en termes de sentiment d'incompétence et d'inefficacité, de dossiers qui demandent beaucoup de temps, de difficulté à demeurer neutre et de départager le vrai du faux et de composer avec un mandat qui manque de clarté. À ce propos, la grande majorité des intervenants $(n=249)$ affirment ressentir toujours $(45 \%)$ ou parfois $(43 \%)$ une pression à prendre position concernant la garde. Lorsqu'ils expliquent leur réponse, les répondants $(n=166)$ apportent des nuances à ce sujet. Certains affirment que la prise de position concernant la garde fait partie de leur mandat quand cette question est reliée à la compromission :

\begin{abstract}
Mon mandat est clair. Le fait que la situation d'un enfant dans ce genre de famille soit compromise ne nécessite pas que nous prenions nécessairement position sur la garde. Parfois, nous laissons les parents décider et intervenons en conséquence pour que la situation ne soit plus compromise. En contrepartie, si l'enfant se retrouve en danger (tant au plan de la sécurité que son développement) dans un milieu contrairement à l'autre, nous pourrions prendre position. (Intervenante 306, évaluation et orientation)
\end{abstract}

D'autres affirment que la pression à prendre position vient surtout des parents, mais aussi parfois du système judiciaire :

De la part des parents, nous le ressentons toujours. Par contre, dans le système judiciaire, nous ne le ressentons pas systématiquement, bien que souvent. C'est apprécié quand nous pouvons nommer nos observations et qu'ils ne nous amènent pas à nous prononcer sur la garde, cela est plus facile pour préserver le lien et poursuivre le travail. (Intervenante 196, application des mesures) 
À ce propos, deux situations sont possibles. D'abord, les intervenants peuvent formuler des recommandations à propos de la garde (par exemple, avec qui l'enfant devrait demeurer, qui il pourra voir et dans quel contexte) devant la Cour du Québec (chambre de la jeunesse) dans le cadre des mesures à mettre en place pour mettre fin à la situation de compromission et uniquement à cette fin. Certaines réponses évoquent également le rôle des intervenants qui sont parfois témoins experts ou témoins de faits en Cour supérieure, cette fois dans des dossiers de séparation et de divorce où la garde est en litige :

Nous sommes souvent interpellés par la Cour supérieure soit en requête de sauvegarde ou requête de changement de garde afin de venir témoigner du meilleur parent à qui on confierait la garde. Les avocats de chacun des parents peuvent aussi nous interpeller à savoir nos motifs d'intervention, le bilan de notre intervention, etc. (Intervenante 137, évaluation et orientation)

Quant au sentiment de devoir à l'occasion sortir de leur mandat dans leurs dossiers comportant des CSS, les intervenants $(n=107)$ rapportent des expériences variées lorsqu'ils répondent à une question ouverte à ce propos. Tel que vu plus tôt, certains sont d'opinion que prendre position sur la garde est dans leur mandat puisque cela est parfois nécessaire pour ne pas porter préjudice à l'enfant, notamment en attendant les délais de la Cour supérieure ou encore quand le jugement de cette même Cour n'est pas respecté, pas assez précis ou ne répond plus au meilleur intérêt de l'enfant. Cependant, la gestion même de ces modalités de garde et des conflits qui les accompagnent pose problème et risque de placer l'intervenant dans un rôle d'intermédiaire ou d'arbitre qui irait à l'encontre de son mandat :

Souvent, bien qu'on est conscients que ça n'est pas dans notre mandat, il est facile de se faire " absorber " par un parent en crise pour une tuque ou une casquette. Qui plus est, lorsque l'enfant est au centre du conflit, il est souvent plus facile de " gérer » les conflits que de les laisser aller. Qui plus est, lorsque nous allons au tribunal, nous sommes questionnés sur l'ensemble de ces conflits et nous nous faisons souvent reprocher par la magistrature de ne pas intervenir pour gérer les conflits. Il est donc souvent difficile, en plus du manque de connaissances sur le traitement des conflits sévères de séparation, de doser les interventions dans l'intérêt de l'enfant, tout en conservant son mandat et en justifiant notre travail devant les tribunaux. (Intervenante 93, application des mesures)

Certains commentaires des intervenants laissent également croire que ces derniers peuvent à l'occasion adopter un rôle s'apparentant à celui d'un médiateur familial :

Nous avons discuté de la garde, pension alimentaire et par la suite les parents ont entériné le tout en Cour supérieure (cela a permis de dénouer certains irritants majeurs dans le conflit), mais est-ce mon mandat? (Intervenante 84, application des mesures) 
Pistes de solutions et d'intervention

À propos des solutions à mettre de l'avant, un certain consensus se dégage chez les 225 intervenants s'étant prononcés sur différentes solutions applicables : une équipe interdisciplinaire ou interprofessionnelle spécialisée en conflits sévères de séparation serait une ressource utile selon 85 \% d'entre eux, le rôle de la protection de la jeunesse devrait être plus clairement défini dans ce type de dossiers (selon $88 \%$ des intervenants) et une formation spécialisée des intervenants serait nécessaire (94\% d'endossement).

Questionnés de façon ouverte à propos des interventions qui ont bien fonctionné par le passé dans leur pratique, des intervenants $(n=194)$ mentionnent l'importance de demeurer neutres notamment dans les moyens de communication avec les parents, de rester centrés sur les besoins de l'enfant sans entrer dans le contenu des conflits, de redonner le pouvoir aux parents et de les responsabiliser, de clarifier le rôle de l'intervenant et de bien cadrer les contacts parent-intervenant dans les limites du mandat des intervenants en protection de la jeunesse. Enfin, des intervenants croient qu'il est nécessaire de travailler ces dossiers en dyade ou en équipe et de ne pas hésiter à consulter les collègues ou supérieurs pour avoir plus de recul sur la situation. Des stratégies inefficaces ont été mentionnées par 170 intervenants et sont, pour plusieurs, l'exact contraire de ce qui a bien fonctionné. Entre autres, il est considéré nuisible d'entrer dans le contenu des conflits (écouter les moindres récriminations et commentaires et tenter de savoir qui dit vrai) et de les gérer à la place des parents. Prendre parti pour l'un des parents serait également un piège à éviter tout comme la confrontation qui apparaîtrait inefficace selon plusieurs.

Quelques contradictions apparaissent également dans l'analyse des réponses à ces deux questions. Si certains promeuvent l'approche systémique et les rencontres familiales, d'autres sont plutôt d'avis qu'il est parfois contre-productif ou prématuré de travailler avec tout le système familial dans une même rencontre. Dans cet ordre d'idées, plusieurs réponses données mentionnent l'approche de médiation instaurée en protection de la jeunesse, notamment dans trois des établissements où la présente recherche s'est déroulée (Chaudière-Appalaches, Montérégie et Québec). Cette méthode alternative de résolution de conflit s'inspire des méthodes développées en médiation familiale, bien qu'elle s'exerce dans un contexte non volontaire et que le " médiateur ", l'intervenant au dossier, ne soit pas un tiers impartial. Elle est cependant caractérisée par la présence simultanée de tous les acteurs (parents, enfants, intervenants et autres personnes significatives) et par l'utilisation de techniques favorisant la communication coopérative. L'autonomie des parties est également valorisée : on les incite à trouver elles-mêmes des solutions pour assurer la sécurité et le développement de l'enfant (Drapeau et al., 2014a; 2014b). Nos 
données qualitatives révèlent que l'approche de médiation est parfois vue par les intervenants comme une bonne approche pour gérer les CSS, mais aussi parfois comme une fausse piste :

Approche médiation, je crois en cette approche, mais dans le cas de conflits sévères, juste asseoir les parents ensemble est un défi. (Intervenante 42, application des mesures)

\section{DISCUSSION}

Cet article avait comme objectif de brosser un premier portrait de la pratique des intervenants en protection de la jeunesse au Québec qui doivent composer avec des situations de CSS et d'identifier les défis et les solutions associés à la prise en charge de ces dossiers. Cette situation apparaît cruciale pour les acteurs du milieu de la protection de la jeunesse, mais aussi pour toute la population du Québec. À ce propos, le bilan 20152016 des DPJ a fortement insisté sur l'importance du dépistage des MTP, ce qui inclut l'exposition des enfants aux CSS, afin de rendre ce problème plus visible et de conscientiser la population à ce "mal silencieux" (INESSS, 2016).

Dans un premier temps, nos résultats confirment le flou existant autour de la définition des CSS qui a déjà été soulevé dans de nombreux travaux (Birnbaum et Bala, 2010; Godbout et al., 2016; Saini et Birnbaum, 2007; Saini et al. 2012). Notamment, la frontière entre les CSS et la violence conjugale reste à clarifier et les intervenants se montrent partagés sur la pertinence d'inclure la violence conjugale dans la définition des CSS. À ce propos, une approche permettant de différencier des formes variées de violence et de mettre en place des plans parentaux sécuritaires pour les enfants et les victimes a été proposée par Jaffe et ses collaborateurs (2008). À notre avis, l'évaluation des CSS comportant des allégations de violence conjugale ne peut faire l'économie de cette évaluation différenciée très rigoureuse considérant le contexte de méfiance et de suspicion concernant les intentions réelles des parents dans ce contexte. En présence de violence conjugale, la séparation est en effet associée à une augmentation de la violence et même à un risque accru de violence extrême, de viol et d'homicide intrafamilial (Jaffe et al., 2008; Lapierre, Lessard et Turcotte, 2004; Comité d'experts sur les homicides intrafamiliaux, 2012).

Malgré la sévérité de ces situations, nos données indiquent que les intervenants de la protection de la jeunesse se sentent très mal outillés pour faire face aux CSS. En effet, ceux-ci n'ayant pas été officiellement définis (ni dans la Loi, ni par les établissements), les intervenants soulèvent des problèmes concernant la clarté de leur mandat quant à la prise en charge de ces dossiers et ils se sentent, par ailleurs, peu formés et sous haute pression (stress élevé, manque de temps, sentiment d'être triangulé dans 
les conflits) pour y faire face. Ces résultats se comparent, à plusieurs égards, à ceux obtenus auprès d'intervenants ontariens (Houston et al., 2017; Saini et al., 2012), laissant présager des défis communs pour les systèmes de protection de la jeunesse au Québec et en Ontario.

Nos résultats révèlent, en outre, qu'une plus vaste expérience des intervenants serait associée à un niveau d'aisance plus élevé dans ces dossiers. Les intervenants masculins rapportent également se sentir plus à l'aise que les intervenantes par rapport aux CSS, et ce, tant au Québec qu'en Ontario (Houston, et al., 2017). Les limites de la méthodologie employée (échantillon de volontaires, absence de contrôle du biais de nonréponse) ne permettent pas de vérifier si l'échantillon recueilli, composé en très forte majorité de femmes ayant 10 ans d'expérience et moins, reflète adéquatement la réalité du terrain. Cependant, si tel est le cas, il pourrait être envisagé que des intervenants ayant plus d'expérience puissent être davantage présents en soutien dans ces cas difficiles. Comme appui à cette idée, les répondants ont identifié le travail en équipe, la co-intervention et le soutien des supérieurs comme stratégies gagnantes dans ces dossiers.

Le mandat des intervenants semble manquer de clarté à différents niveaux, notamment concernant les contextes précis où ils peuvent ou doivent se positionner sur la garde (notamment devant la Cour du Québec et parfois devant la Cour supérieure) et s'il est opportun ou non d'aider les parents à trouver des ententes ou de gérer les conflits quotidiens de ces derniers. Leur rôle régi par les limites imposées par la Loi est également souvent mal compris par des intervenants externes dans ces dossiers. Par ailleurs, les intervenants semblent trouver ardu de faire la preuve de la compromission de la sécurité et du développement dans les dossiers de CSS et rapportent avoir reçu peu de directives de leur établissement pour ces cas particuliers. Pour toutes ces raisons, il semble indiqué de travailler à établir une approche commune qui clarifierait les rôles, les responsabilités et le mandat de la DPJ et de ses intervenants en présence de CSS. Les intervenants pourraient ainsi mieux résister aux pressions qu'ils disent ressentir de tous les côtés, que ce soit de la part des parents, des professionnels externes et même des tribunaux.

Des réponses au présent sondage indiquent que les intervenants sont parfois interpellés par la Cour supérieure, principalement lors de litiges de garde et de droits d'accès. Les données disponibles à ce sujet montrent que ces interventions seraient plutôt rares en Ontario (Houston, et al., 2017). À notre connaissance, aucune publication n'a dénombré ou ne documente ces interventions à la Cour supérieure du Québec. Dans cet ordre d'idées, les conséquences des nouvelles compétences accordées à la Cour du Québec (concernant notamment la garde des enfants) dans les dossiers de protection de la jeunesse dont cette Cour est déjà saisie mériteraient d'être 
observées de près. II s'agit là d'occasions de repenser le traitement judiciaire de ces dossiers pour éviter les problèmes de coordination ou les décisions incohérentes, les délais indus et pouvant porter préjudice à l'enfant et la perte de temps et de ressources financières.

Sur le plan des solutions pour soutenir l'intervention en protection de la jeunesse, l'approche de médiation déjà bien intégrée aux services semble parfois utile, parfois contre-productive selon les participants à l'étude. Les intervenants interrogés par Drapeau et ses collaborateurs (2014a) ont fait état de la même contradiction à propos de cette approche qui est vue, dans les dossiers de CSS et d'aliénation parentale, à la fois comme l'approche idéale dans certains cas et comme une approche inefficace dans des situations jugées trop complexes et rigidifiées. Un parallèle est ici à faire avec la médiation familiale qui s'avère souvent inefficace dans les cas de séparations hautement conflictuelles très cristallisées (Cyr, Poitras, Godbout et Macé, 2017). Ces résultats soulignent la nécessité de développer un triage efficace de ces situations et une approche différenciée dans l'intervention afin d'individualiser la réponse à apporter à chaque cas particulier (Fidler, Bala et Saini, 2011). Certains services psychosociaux et judiciaires devraient ainsi être réservés aux familles ayant des conflits plus modérés ( $p$. ex., médiation familiale, droit collaboratif), d'autres services devraient mieux convenir aux familles vivant des conflits plus persistants ( $p$. ex., médiation familiale thérapeutique et spécialisée, thérapie familiale multiniveaux, programmes éducatifs spécialisés) et les interventions visant la protection devraient, quant à elles, être bien encadrées par des ordonnances et un suivi judiciaire continu dans les cas les plus sévères de perte de lien parent-enfant ou encore lorsque la sécurité est un enjeu (p. ex., coordination parentale, supervision des droits d'accès, protocole d'intervention psychojudiciaire) (Cyr et al., 2017; Godbout et al., 2017; Johnston et al., 2009). II convient cependant de souligner que la recherche sur les interventions à privilégier auprès de ces familles en fonction des degrés de sévérité mentionnés plus tôt en est à ses premiers balbutiements.

Des initiatives récentes (projets pilotes et guides pour la pratique) sont en cours afin de mieux répondre aux besoins spécifiques de ces familles et pour soutenir l'intervention (Charette, 2018; Cyr et al., 2017; Godbout et al., 2017). L'état actuel des connaissances et le développement de la pratique ne sont toutefois pas encore en phase avec la réalité du terrain qui se fait pressante. Les prochaines années seront donc cruciales pour mieux comprendre les CSS et unir nos efforts pour répondre adéquatement à cet important problème.

\section{RÉFÉRENCES}

Amato, P. R. (2003). Reconciling divergent perspectives: Judith Wallerstein, quantitative family research, and children of divorce. Family Relations, 52(4), 332-339. 
Amato, P. R. et Keith, B. (1991). Parental divorce and the well-being of children: A metaanalysis. Psychological Bulletin, 110(1), 26-46.

Anderson, S. R., Anderson, S. A., Palmer, K. L., Mutchler, M. S. et Baker, L. K. (2010). Defining high conflict. The American Journal of Family Therapy, 39(1), 11-27.

Bala, N., Birnbaum, R. et Martinson, J. D. (2010). One judge for one family: Differentiated case management for families in continuing conflict. Canadian Journal of Family Law, 26(2), 395-450.

Bala, N. et Houston, C. (2015). The challenge of high-conflict family cases involving a child protection agency: A review of literature and an analysis of reported Ontario cases. Queen's University Legal Research Paper, 84.

Barreau du Québec (2013). Mémoire du Barreau du Québec sur le projet de loi no 28 intitulé Loi instituant le nouveau Code de procédure civile. Récupéré le $1^{\text {er }}$ février 2018 de https://www.barreau.qc.ca/pdf/medias/positions/2013/20130913-pl-28.pdf

Biland, É. et Schütz, G. (2012). Pour avoir la garde, il faut la demander! Les déterminants de la garde des enfants dans les procédures judiciaires au Québec. Conférence présentée au 2 e colloque franco-québécois du projet de recherche Ruptures. Les séparations conjugales : décisions judiciaires et incidences familiales, Université de Montréal.

Biland, É. et Schütz, G. (2013). La garde des enfants de parents séparés au Québec : une analyse quantitative de dossiers judiciaires. Collection Que savons-nous? Québec: ARUC-séparation parentale, recomposition familiale.

Birnbaum, R. et Bala, N. (2010). Towards the differentiation of high-conflict families: An analysis of social science research and Canadian case law. Family Court Review, 48(3), 403-416.

Black, T., Saini, M., Deljavan, S., Fallon, B., Theoduloz, R et Wall, M. (2016). The intersection of child custody disputes and child protection investigations: Secondary data analysis of the Canadian Incidence Study of Reported Child Abuse and Neglect (CIS-2008). International Journal of Child and Adolescent Resilience, 4(1), 143-157.

Brown, T. (2003). Fathers and child abuse allegations in the context of parental separation and divorce. Family Court Review, 41(3), 367-380.

Brown, T., Frederico, M., Hewitt, L. et Sheehan, R. (2000). Revealing the existence of child abuse in the context of marital breakdown and custody and access disputes. Child Abuse \& Neglect, 24(6), 849-859.

Brown, T., Frederico, M., Hewitt, L. et Sheehan, R. (2001). The child abuse and divorce myth. Child Abuse Review, 10(2), 113-124.

Buckley, H., Whelan, S. et Carr, N. (2011). 'Like waking up in a Franz Kafka novel': Service users' experiences of the child protection system when domestic violence and acrimonious separations are involved. Children and Youth Services Review, 33(1), 126-133.

Buehler, C., Anthony, C., Krishnakumar, A., Stone, G., Gerard, J. et Pemberton, S. (1997). Interparental conflict and youth problem behaviors: A meta-analysis. Journal of Child and Family Studies, 6(2), 233-247.

Charette, L. (2018). Le traitement des conflits sévères de séparation: enjeux et défis du système judiciaire. Conférence organisée par le Laboratoire de psychologie légale de l'Université du Québec à Trois-Rivières, Trois-Rivières, Québec.

Comité de réflexion et d'orientation sur la justice de première instance au Québec. (2005). Une réforme judiciaire axée sur le citoyen. Cour du Québec. Récupéré le $1^{\text {er }}$ février $2018 \mathrm{de}$ http://www.tribunaux.qc.ca/cquebec/CommuniquesDocumentation/reformeJudiciaireCitoyen.pdf

Comité d'experts sur la révision de la Loi sur la protection de la jeunesse. (2004). La protection des enfants au Québec : une responsabilité à mieux partager. Québec, Québec : Direction générale des services à la population du ministère de la Santé et des Services sociaux.

Comité d'experts sur les homicides intrafamiliaux (2012). Rapport du comité d'experts sur les homicides intrafamiliaux. Québec, Québec : Ministère de la Santé et des Services sociaux. Récupéré de http://publications.msss.gouv.qc.ca/msss/fichiers/2012/12-803-02.pdf

Conseil de la famille et de l'enfance (2003). L'allègement du processus judiciaire en matière familiale. Mieux soutenir les parents et les enfants lors des contentieux familiaux. Québec, Québec: Ministère de la Famille et de l'Enfance.

Cummings, E. M. et Davies, P. (1994). Children and marital conflict: The impact of family dispute and resolution. New York, NY : Guilford Press. 
Cyr, F., Poitras, K., Godbout, E. et Macé, C. (2017). Évaluation du protocole de gestion psychojudiciaire axé sur la parentalité, les conflits et leur résolution (PCR). Rapport de recherche, ministère de la Justice du Québec.

Deljavan, S., Saini, M., Fallon, B. et Black, T. (2016). The intersection of child custody disputes and children's exposure to intimate partner violence. Affiche présentée au $53^{\mathrm{e}}$ congrès annuel de l'AFCC, Modern Families: New Challenges, New Solutions, Seattle, WA.

Desrosiers, H. et Simard, M. (2010). Diversité et mouvance familiales durant la petite enfance Étude longitudinale du développement des enfants du Québec (ÉLDEQ 1998-2010) - De la naissance à 6 ans (vol. 4). Québec, Québec : Institut de la statistique du Québec.

Drapeau, S., Tremblay, J., Cyr, F., Godbout, E. et Gagné, M.-H. (2008). La coparentalité chez les parents séparés. Dans C. Parent, S. Drapeau, M. Brousseau et E. Pouliot (dir.) Visages multiples de la parentalité (p. 255-281), Québec, Québec: Presses de l'Université du Québec.

Drapeau, S., Tremblay, J., Lessard, G., Turcotte, D., Mireault, G. et Gagné, M.-H. (2014a). Application d'une approche de médiation en protection de la jeunesse : qu'en pensent les intervenants? Service social, 60(2), 14-28.

Drapeau, S., Tremblay, J., Turcotte, D., Lessard, G., Lemieux, R. et Mireault, G. (2014b). L'approche de médiation en protection de la jeunesse : une voie vers des interventions plus consensuelles? Intervention, 140, 29-36.

Emery, R. E. (1999). Marriage, divorce, and children's adjustment. Thousand Oaks, CA : Sage Publications.

Fauteux, M.-H. (2013). Les mauvais traitements psychologiques caractérisés par des conflits entre les parents en contexte de Centre Jeunesse. Mémoire doctoral en psychologie, Université Laval.

Fidler, B. J., Bala, N. et Saini, M. (2011). Ten tips for legal and mental health professionals involved in alienation, or alleged alienation. Association of Family and Conciliation Courts Monthly E-Newsletter, 6(5). Repéré à https://www.afccnet.org/Portals/0/PDF/ Ask_the_Experts_Book.pdf

Friedman, M. (2004). The so-called high-conflict couple: A closer look. The American Journal of Family Therapy, 32(2), 101-117.

Gagné, M.-H. (2001). Les pratiques parentales psychologiquement violentes : une menace à la santé mentale. Revue canadienne de santé mentale communautaire, 20(1), 75-106.

Gagné, M. H. et Bouchard, C. (2000). Validation sociale du construit: "violence psychologique » dans le cas des enfants maltraités. Revue canadienne de service social, 17(2), 225-244.

Gagné, M.-H., Cyr, F., Drapeau, S., Saint-Jacques, M.-C. et Lessard, G. (2011). Les manifestations d'aliénation parentale dans les situations évaluées en protection de la jeunesse. Communication présentée lors des Journées de la recherche du CJQ-IU, Québec, Québec.

Godbout, E., Lachance, V., Pauzé, R., Baude, A., Gauthier, M., Groleau, H. et Noël, J. (2017). Recension des écrits sur les facteurs de risque associés aux conflits sévères de séparation et sur les conséquences possibles sur l'adaptation des enfants. Outil destiné aux intervenants du CIUSSS de la Capitale-Nationale.

Godbout, E., Parent, C. et Saint-Jacques, M.-C. (2016). Les séparations hautement conflictuelles et le meilleur intérêt de l'enfant vu de l'intérieur : analyse du point de vue des experts et des juges. Dans M.-C., Saint-Jacques, C. Robitaille, A. St-Amand et S. Lévesque (dir.), Séparation parentale, recomposition familiale: Enjeux contemporains (p. 187-210). Québec, Québec : Presses de l'Université du Québec.

Grych, J. H. (2005). Interparental conflict as a risk factor for child maladjustment: implications for the developement of Prevention Programs. Family Court Review, 43(1), 97-108.

Hetherington, E. M., Bridges, M. et Insabella, G. M. (1998). What matters? What does not? Five perspectives on the association between marital transitions and children's adjustment. American Psychologist, 53(2), 167-184.

Hetherington, E. M. et Stanley-Hagan, M. (1999). The adjustment of children with divorced parents: A risk and resiliency perspective. Journal of Child Psychology and Psychiatry, $40(1), 129-140$. 
Houston, C., Bala, N. et Saini, M. (2017). Crossover cases of high-conflict families involving child protection services: Ontario research findings and suggestions for good practices. Family Court Review, 55(3), 362-374.

Institut national d'excellence en santé et en services sociaux (INESSS) (2016). Les mauvais traitements psychologiques : un mal silencieux. Bilan des directeurs de la protection de la jeunesse 2016. Récupéré de https://www.inesss.qc.ca/fileadmin/doc/INESSS ServicesSociaux/Bilan_DPJ/INESSS_Bilan_DPJ_Mauvais_traitements_psycho_2016.pdf

Jaffe, P. G., Johnston, J. R., Crooks, C. V. et Bala, N. (2008). Custody disputes involving allegations of domestic violence: toward a differentiated approache to parenting plans. Family Court Review, 46(3), 500-522.

Jevne, K. S. et Andenæs, A. (2017). Parents in high-conflict custodial cases: negotiating shared care across households. Child \& Family Social Work, 22(1), 296-305.

Johnson, M. P. (2008). A typology of domestic violence: intimate terrorism, violent resistance and situational couple violence. Boston, MA : Northeastern University Press.

Johnston, J. R. (1994). High-conflict divorce. The Future of Children, 4(1), 165-182.

Johnston, J. R. (2006). A child-centered approach to high-conflict and domestic-violence families: Differential assessment and interventions. Journal of Family Studies, 12(1), 1535.

Johnston, J. R., Lee, S., Olesen, N. W. et Walters, M. G. (2005). Allegations and substantiations of abuse in custody disputing families. Family Court Review, 43(2), 283-294.

Johnston, J. R., Roseby, V. et Kuehnle, K. (2009). In the name of the child: A developmental approach to understanding and helping children of conflicted and violent divorce. New York, NY : Springer.

Joyal, R., Lapierre-Adamcyk, É., Le Bourdais, C. et Marcil-Gratton, N. (2002). Le rôle des tribunaux dans la prise en charge des enfants après le divorce ou la séparation des parents. Québec, Québec : Ministère de l'Emploi, de la Solidarité sociale et de la Famille du Québec.

Kelly, J. B. (2000). Children's adjustment in conflicted marriage and divorce: A decade review of research. Journal of the American Academy of Child \& Adolescent Psychiatry, 39(8), 963-973.

Kelly, J. B. (2003). Parents with enduring child disputes: Multiple pathways to enduring disputes. Journal of Family Studies, 9(1), 37-50.

Lapierre, S., Lessard, G. et Turcotte, D. (2004). La séparation dans un contexte de violence conjugale : quels sont les défis pour l'intervention auprès des enfants? Dans M.-C. SaintJacques, D. Turcotte, S. Drapeau et R. Cloutier (dir.), Séparation, monoparentalité et recomposition familiale. Bilan d'une réalité complexe et pistes d'action (p. 195-216). Québec, Québec : Presses de l'Université Laval.

Levite, Z. et Cohen, O. (2012). The tango of loving hate: Couple dynamics in high-conflict divorce. Clinical Social Work Journal, 40(1), 46-55.

Maccoby, E. E. et Mnookin, R. H. (1992). Dividing the child: Social and legal dilemmas of custody. Cambridge, MA : Harvard University Press.

Malo, C. (2013). L'exposition au conflit sévère de séparation : ce qui la distingue de l'aliénation parentale. Séminaire présenté à l'Association des Médecins en Protection de l'Enfance du Québec.

Malo, C. (2014). Coup d'œil sur les mauvais traitements psychologiques. Site internet de l'Observatoire sur la maltraitance envers les enfants. Récupéré de http://observatoiremaltraitance.ca/Pages/Coup-d'oeil-sur-les-mauvais-traitementspsychologiques.aspx/

Malo, C. et Rivard, D. (2013). Aliénation parentale et exposition aux conflits sévères de séparation: Où en sommes-nous? Montréal, Québec: Centre jeunesse de MontréalInstitut universitaire.

Ministère de la Santé et des Services sociaux (2017). Bilan annuel des directeurs de la protection de la jeunesse. Récupéré de https://www.cisss-ca.gouv.qc.ca/fileadmin/ documents/Services_offerts/Enfants-Jeunes-Famille/Bilan_2016-2017.pdf

Noller, P., Feeney, J. A., Sheehan, G., Darlington, Y. et Rogers, C. (2008). Conflict in divorcing and continuously married families: a study of marital, parent-child and sibling relationships. Journal of Divorce \& Remarriage, 49(1-2), 1-24 
Paillé, P. et Mucchielli, A. (2008). L'analyse qualitative en sciences humaines et sociales ( $2^{\mathrm{e}}$ éd.). Paris, France : Armand Colin.

Perron, É. B. et Labrecque Tremblay, J. (2015). Garde d'enfant et autorité parentale en Cour du Québec, Chambre de la jeunesse: comme familialiste, à quoi s'attendre? Dans Barreau du Québec (dir.), Développements récents en droit familial (p 19-51). Montréal, QC : Éditions Yvon Blais.

Platt, S., Ajami, J., Kluemper, N., Geffner, R., Shaw, M. et Assalley, A. (2016). Child protection in child custody cases: Issues and concerns. Dans S. Deb (dir.), Child safety, welfare and well-being: Issues and challenges (p. 313-333). New Delhi, Inde : Springer India.

Poitras, K., Cyr, F. et Chatigny, A. (accepté). Trajectoires psychojudiciaires suite à la séparation parentale : études de dossiers en provenance du Québec. Revue scientifique de l'AIFI.

Saini, M. A. et Birnbaum, R. (2007). Unraveling the label of "high conflict": What factors really count in separated and divorced families? Part 1. Ontario Association of Children's Aid Societies Journal, 51(1), 14-29.

Saini, M. A., Black, T., Fallon, B. et Marshall, A. (2013). Child custody disputes within the context of child protection investigations: Secondary analysis of the Canadian Incident Study of Reported Child Abuse and Neglect. Child Welfare, 92(1), 115-137.

Saini, M., Black, T., Lwin, K., Marshall, A., Fallon, B. et Goodman, D. (2012). Child protection worskers'experiences of working with high-conflict separating families. Children and Youth Services Review, 34(7), 1309-1316.

Turbide, C. et Saint-Jacques, M.-C. (2017). Comprendre le conflit sévère de séparation : et si on tenait compte du contexte? Communication présentée lors du colloque : "Séparation parentale, recomposition familiale : enjeux contemporains ", $85^{\mathrm{e}}$ Congrès de l'ACFAS, Montréal (Université McGill).

\section{RÉSUMÉ}

Les familles aux prises avec des conflits sévères de séparation (CSS) représentent un défi de taille pour les services de protection de la jeunesse. Cet article rend compte de l'expérience d'intervenants québécois $(n=309)$ dans ce type de dossiers à l'aide d'un sondage en ligne. Les résultats révèlent que cette problématique est mal définie selon les intervenants et que le mandat de la protection de la jeunesse manque de clarté dans ces dossiers. Une approche à la fois intégrée et différenciée des services psychojudiciaires est discutée pour le développement futur de l'intervention dans ce domaine.

\section{MOTS CLÉS}

protection de la jeunesse, conflits sévères de séparation, séparations hautement conflictuelles, conflits de garde et de droits d'accès

\section{ABSTRACT}

Families involved in high conflict post separation pose unique challenges for child protection services. This study is based on an online survey of Quebec child protection workers' $(n=309)$ experience with high conflict situations. Results show that child protection cases with high conflict families are complicated by workers' lack of operational definition of high conflict situations and by an unclear mandate of child protection services. Implications for practice include developing both an integrated approach and a differential response to better respond to families involved in high conflict. 
Les conflits sévères de séparation

KEY WORDS

child protection; high conflict, child custody and access dispute 